\title{
O SISTEMA APACHE II E O PROGNÓSTICO DE PACIENTES SUBMETIDOS ÀS OPERAÇÕES DE GRANDE E PEQUENO PORTE
}

\author{
APACHE II SCORES AND PROGNOSIS FOR PATIENTS SUBMITTED TO MAJOR AND \\ MINOR SURGERIES
}

\author{
Miguel Angelo Martins de Castro Junior, TCBC-RS²; Miguel Angelo Martins de Castro, TCBC-RS²; \\ Angela Potter de Castro ${ }^{3}$; Alcino Lázaro da Silva
}

\begin{abstract}
RESUMO: Objetivo: Geral: avaliar o desempenho do sistema APACHE II no pré-operatório de operações de grande e pequeno porte como indicador prognóstico. Os objetivos secundários são: (a) avaliar o sistema no pré-operatório para predizer morbidade; (b) comparar resultados entre os dois grupos e; (c) comparar a mortalidade observada com a esperada, calculada pela Equação de Knaus. Método: Estudo de coorte prospectivo, por 30 meses. No Grupo 1, correspondente à operação de pequeno porte, incluídos 30 pacientes submetidos a herniorrafia inguinal. No Grupo 2, correspondente à operação de grande porte, incluídos 50 casos, sendo: 20 submetidos à esofagectomia, 20 à gastrectomia e 10 à duodenopancreatectomia cefálica. Resultados: A média do APACHE II no Grupo 1 foi de 2,5 pontos ( 0 a 8 pontos).No Grupo 2 teve média de 3,9 pontos ( 0 a 12 pontos). A morbidez tanto geral quando calculada de acordo com índice de pontos baixo, moderado e alto, apresentaram significância na comparação de ambos grupos pelo teste quiquadrado $(\mathrm{p}<0,00001)$. A mortalidade geral e o índice alto de pontos, no cálculo quiquadrado corrigido Yates, apresentaram diferença entre os grupos $(p<0,001$ e $p<0,0005)$. Estratificada a mortalidade por classe de pontos, esta não foi diferente nas classes de baixa e moderada pontuação. Conclusão: $O$ sistema demonstrou tendência para predizer desfecho a pacientes submetidos à operação de grande porte. Pacientes apresentando, no pré-operatório de operações de grande porte, APACHE II acima de oito apresentam índice elevado de morbidez e mortalidade (Rev. Col. Bras. Cir. 2006; 33(5): 272-278).
\end{abstract}

Descritores: APACHE; Prognóstico; Cuidados pré-operatórios; Complicações pós-operatórias.

\section{INTRODUÇÃO}

A necessidade de respostas sobre como o doente se apresentará após ter sido submetido a uma injúria, deparase com o processo de reconhecimento da multifatoriedade e complexidade do organismo. Seria possível inseri-lo em uma escala, índice, escore, que teria o objetivo de predizer prognóstico ou morbidez, e ainda, independente do seu diagnóstico, forma de apresentação, evolução, tratamentos prévios, iatrogênicos ou não?

A avaliação da morbimortalidade é um dos alicerces que regem a escolha terapêutica. Tentar classificar o paciente em valores numéricos e expressivos seria o ideal e vantajoso. A primeira tentativa foi realizada por especialistas em Anestesia na década de 60 (Classificação da ASA) ${ }^{1}$.

O desenvolvimento de novas formas de tratamento, da anestesia, das unidades de tratamento intensivo, tornou possível a realização de grandes procedimentos cirúrgicos e a recuperação de pacientes que anteriormente apresentavam o óbito como desfecho. O cirurgião tem o conhecimento terapêutico e fisiopatológico do trauma cirúrgico e suporte para recuperação, porém fica a dúvida de qual paciente se beneficiará. O conhecimento da estatística e da medicina baseada em evidência trouxe o suporte necessário para uma nova era de índices matematicamente comprovados como eficazes.

A escala denominada "Acute Physiology And Chronic Health Evaluation - APACHE” foi desenvolvida por Knaus ${ }^{2}$ em 1981 e revisada e simplificada em $1985^{3}$. O método tem o intuito de responder à possibilidade de recuperação. Devido à facilidade e mensurabilidade de sua utilização, e mais importante, aos seus resultados com base estatística, houve extrapolação na sua utilidade.

Bohnen $^{4}$ desenvolveu o primeiro trabalho prospectivo e de relevância científica nesse método e comprovou que o APACHE II poderia ser utilizado para predizer sobrevida no pré-operatório $(\mathrm{p}<0,05)$ em pacientes acometidos por sepse abdominal. Nem todos os estudos são totalmente conclusivos, como Cerra $^{5}$ demonstrou em seu artigo, que disserta sobre o desenvolvimento de insuficiência de

1. Professor Assistente do Departamento de Cirurgia da Fundação Universidade Federal do Rio Grande - FURG, Rio Grande, RS; Mestre em Medicina pela Universidade Federal do Rio Grande do Sul - UFRGS.

2. Professor Titular do Departamento de Cirurgia da Fundação Universidade Federal de Rio Grande - FURG, Rio Grande, RS; Doutor em Medicina pela Universidade Federal de Minas Gerais - UFMG.

3. Professora Substituta do Departamento de Cirurgia da Fundação Universidade Federal de Rio Grande - FURG; Mestre pela Universidade Federal do Rio Grande do Sul - UFRGS.

4. Professor Titular do Departamento de Cirurgia da Faculdade de Medicina da Universidade Federal de Minas Gerais, Belo Horizonte, MG.

Recebido em 30-03-2006

Aceito para publicação em 02-05-2006

Conflito de interesses: nenhum

Fonte de financiamento: nenhuma

Trabalho realizado no Serviço de Cirurgia Torácica do Hospital Nossa Senhora da Conceição, Porto Alegre, RS, e Departamento de Cirurgia da Fundação Universidade Federal de Rio Grande, Rio Grande, RS. 
múltiplos órgãos e sistemas (IMOS) e óbito, em que chega a conclusão que o APACHE II falhou em revelar este desfecho.

O objetivo geral deste trabalho é avaliar o desempenho do escore APACHE II no pré-operatório dos procedimentos cirúrgicos de grande e pequeno porte como indicador prognóstico.

Os objetivos secundários são: (a) avaliar o escore no pré-operatório para predizer morbidade; (b) comparar resultados entre os dois grupos e; (c) comparar mortalidade observada com esperada calculada por Equação de Knaus ${ }^{3}$.

\section{MÉTODO}

O delineamento foi o estudo de coorte prospectivo, com coleta de dados por um período de 30 meses, sendo os pacientes acompanhados até sua alta ou óbito. Previamente revisado e aprovado pelo Comitê de Ética.

A casuística foi dividida em dois grupos. No Grupo 1 (operação de pequeno porte), foram incluídos 30 pacientes submetidos à herniorrafia inguinal. No Grupo 2 (operação de grande porte), foram incluídos 50 pacientes, sendo: 20 submetidos à esofagectomia, 20 à gastrectomia e 10 à duodenopancreatectomia cefálica.

A análise dos dados incluiu revisão pré-operatória dos prontuários dos pacientes. O escore APACHE II foi calculado de acordo com o método de Knaus ${ }^{3}$. Para cada variável fisiológica, foi valorizada a maior alteração mensurada 24 horas antes da cirurgia. Em algumas situações de exceção na qual a variável não tinha sido medida, o último valor obtido no pré-operatório foi utilizado em seu lugar. No caso da variável não existir, ela foi considerada normal, e zero ponto foi-lhe dada. Uma similar atribuição de pontos foi validada em outros estudos ${ }^{4,6}$.

O protocolo utilizado, além do escore APACHE II, registrava ainda: etiologia, sexo, etnia, técnica cirúrgica, dias de internação, morbidez e óbitos. A morbidez foi definida como qualquer complicação ocorrendo da admissão do paciente até 30 dias após o ato operatório. O óbito ocorrendo em 30 dias após a cirurgia ou durante a internação foi definido como morte operatória. O tempo de hospitalização foi definido como o tempo de permanência no hospital desde a internação até o momento em que o paciente recebeu alta pela equipe cirúrgica ou morreu.

Os pacientes do Grupo 1 foram submetidos à herniorrafia inguinal, conforme a técnica de preferência do cirurgião titular, sendo elas: reparo de Bassini ${ }^{7}$; reparo de $\mathrm{MacVay}^{7}$; reparo de Lichtenstein ${ }^{7}$.

No Grupo 2 os doentes esofagectomizados foram

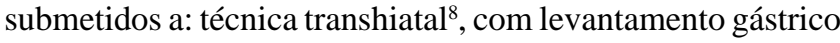
pelo mediastino posterior e anastomose cervical; técnica transtorácica ${ }^{8}$, com toracotomia direita e dissecção esofágica, fechamento desta abordagem, e subsequente esqueletização do estômago e levantamento gástrico pelo mediastino posterior com anastomose cervical. Os doentes gastrectomizados ou eram submetidos à gastrectomia subtotal ${ }^{9}$ ou à total ${ }^{10} \mathrm{com}$ reconstrução através da técnica de Y de Roux. Os doentes submetidos a duodenopancreatectomia ${ }^{11}$ foram conduzidos com ressecção da lesão de forma habitual que incluía também antrectomia. A reconstrução fez-se com anastomose pancreatojejunal términoterminal com telescopagem, hepaticojejunoanastomose terminolateral e gastroenteroanastomose terminolateral do tipo Polya9 .

Todas as operações foram realizadas na abordagem padrão, não laparoscópico. Os procedimentos cirúrgicos foram realizados por duas equipes de cirurgiões gerais.

A avaliação prognóstica é baseada na seguinte regressão logística, proveniente do trabalho inicial do escore APACHE II de Knaus ${ }^{3}: \mathrm{R}=-3,517$ + (APACHE II x 0,146) 0,248 . Onde o valor de -0,248 é a variável para operação digestiva por neoplasia, segundo Knaus ${ }^{3}$.

Os pacientes foram divididos em três grupos de acordo com o resultado de APACHE II no pré-operatório: baixo, 0 a 3 pontos; moderado, 4 a 7 pontos; e alto, maior que oito pontos $^{12}$

Os resultados foram codificados e depois armazenados e analisados no programa Epi Info versão 6.04b. Realizouse, inicialmente, uma análise descritiva da amostra. Resultados quantitativos são demonstrados com desvio-padrão e foram utilizados os testes " $t$ " Student, analise da variância, e teste exato de Fisher para a análise estatística. O valor de $\mathrm{p}<0,05$ foi considerado significante e o intervalo de confiança de $95 \%$. De acordo com o nível do APACHE foi estabelecido o risco relativo.

\section{RESULTADOS}

No Grupo 1 em relação ao sexo, foram $87 \%$ pacientes masculinos e $13 \%$ femininos. A idade média foi de 48 anos (variando de 18 a 90 anos), 97\% eram brancos. A técnica de preferência foi de MacVay, em $40 \%$ dos pacientes seguido de Bassini em $33 \%$ e Lichtenstein em $27 \%$.

Apenas um paciente submetido ao reparo de Mac $V{ }^{7}$, apresentou complicação pós-operatória desenvolvendo edema escrotal, com resolução espontânea em dias. A média de internação foi de 2,2 dias (variando de um a cinco dias). Não houve óbitos. A distribuição em relação ao escore: baixo: 22 pacientes; moderado: sete pacientes; e alto: um paciente. A média do escore APACHE II destes doentes foi de 2,5 pontos (variando de 0 a 8 pontos), com desvio padrão de 2,2 pontos.

No Grupo 2 em relação ao sexo, foram $56 \%$ masculinos e $44 \%$ femininos. A idade média foi de 58,7 anos (variando de 36 a 84 anos). Considerando os tipos de operações a idade média foi: duodenopancreatectomia com 58,4 anos (variando de 46 a 68 anos); gastrectomia com 61,3 anos (variando de 36 a 84 anos) e esofagectomia com 56,2 anos (variando de 37 a 76 anos), 94\% eram leucodérmicos. O diagnóstico destes é visto na Tabela 1. Os procedimentos cirúrgicos são vistos na Tabela 2 .

A mortalidade Geral do grupo 2 foi de $24 \%$ e distribuindo-se por tipo de operação: duodenopancreatectomia (DPT) com quatro casos $(40 \%)$; gastrectomia com quatro casos $(20 \%)$; e esofagectomia com quatro casos $(20 \%)$.

Ainda neste grupo, a morbidade geral foi de $72 \%$, dividida em (34\%) complicações maiores e $(66 \%)$ menores (Tabela 3 ). 
Tabela 1 - Diagnóstico etiológico.

\begin{tabular}{llrr}
\hline & Diagnóstico & Casos & $\mathbf{( \% )}$ \\
\hline ESOFAGECTOMIA & Carcinoma Epidermóide de Esôfago & 17 & $(35)$ \\
GASTRECTOMIA & Adenocarcinoma de Esôfago & 3 & $(5)$ \\
& Adenocarcinoma Gástrico & 18 & $(36)$ \\
DUODENOPANCREATECTOMIA & Linfoma Gástrico & 1 & $(2)$ \\
& Tumor Carcinóde Gástrico & 1 & $(2)$ \\
& Adenocarcinoma Cabeça Pâncreas & 8 & $(16)$ \\
& Tumor Carcinóide Papila Vater & 1 & $(2)$ \\
& Adenocarcinoma Papila de Vater & 1 & $(2)$ \\
\hline
\end{tabular}

Tabela 2 - Operação utilizada nos pacientes do Grupo 2.

\begin{tabular}{llrr}
\hline Operação & & Casos & $\mathbf{( \% )}$ \\
\hline ESOFAGECTOMIA & Transhiatal (levantamento Gástrico) & 7 & $(14)$ \\
& Transhiatal (levantamento Cólon) & 1 & $(2)$ \\
& Transtorácica (levant. Gástrico) & 11 & $(22)$ \\
GASTRECTOMIA & Transtorácica (levantamento Cólon) & 1 & $(2)$ \\
& Gastrectomia Total & 8 & $(16)$ \\
DUODENOPANCREATECTOMIA & Gastrectomia Parcial & 12 & $(24)$ \\
& Técnica de Whipple & 10 & $(20)$ \\
\hline
\end{tabular}

A média dos dias de internação do grupo de cirurgia de grande porte foi de 35 dias, variando de 11 a 99 dias. Os submetidos a DPT, gastrectomia e esofagectomia tiveram média de internação de 35 dias, variando de 11 a 61 dias, 11 a 72 dias e 11 a 99 dias, respectivamente.

O escore APACHE II no Grupo 2 teve a média de 3,9 pontos, variando de zero a 12 pontos (desvio padrão: 2,8 pontos). Em relação à cirurgia: a DPT teve a média de quatro pontos, variando de dois a nove pontos (desvio padrão: 2,3 pontos); a gastrectomia teve a média de 4,8 pontos, variando de zero a 12 pontos (desvio padrão: 3,5 pontos); a esofagectomia teve média de 3,1 pontos, variando de 0 a 6 pontos (desvio padrão: 1,8 pontos).

Os resultados de morbidez e mortalidade do Grupo 2 são apresentados na Tabela 4. A morbidez aumentou com o aumento do escore, principalmente comparando classes de escore baixo e moderado com o APACHE maior e igual a oito, demonstrando uma tendência, porém nenhumas destas diferenças foram estatisticamente significantes. Esta tendência foi comprovada $(\mathrm{p}<0,04)$ quando comparada a mortalidade da classe de escore alto com o de baixo escore pelo teste exato de Fischer. O risco relativo apresentado para evoluir para o óbito foi de 5,4, com intervalo de confiança que variou de 1,49 a 19,51. Em relação ao escore moderado, foi demonstrada tendência, porém não significativo $(\mathrm{p}<0,08)$.

Os Grupos 1 e 2 foram comparados nos seguintes dados: dias de internação, média de APACHE e morbimortalidade.

As variáveis, dias de internação e média do APACHE, apresentaram diferença estatística na análise da variância da média quando comparados os dois grupos $(\mathrm{p}<0,00001 \mathrm{e} p<0,05$, respectivamente).
A morbidez tanto geral quanto a calculada de acordo com índice de pontos baixo, moderado e alto, apresentaram significância na comparação de ambos grupos pelo teste quiquadrado $(\mathrm{p}<0,00001)$.

A mortalidade geral e o índice alto de pontos, no cálculo quiquadrado corrigido Yates, apresentaram diferença entre os grupos $(\mathrm{p}<0,001 \mathrm{e} \mathrm{p}<0,0005)$. Quando estratificada a mortalidade por classe de pontos, esta não foi diferente estatisticamente nas classes de baixa e moderada pontuação.

Quando se analisa os resultados do APACHE II geral em relação à sua média para sobreviventes e óbitos no Grupo 2 , evidencia-se que os pacientes que evoluíram para óbito apresentaram média de APACHE de 5,9 pontos comparado aos sobreviventes que foi de 3,3. A diferença entre estas duas mensurações foi estatisticamente significativa ( $p<0,01)$. Quando esta comparação é estratificada para os três tipos de ressecção cirúrgica, apenas os pacientes submetidos a duodenopancreatectomia apresentaram diferença estatística ( $\mathrm{p}<0,03)$, sendo a média do APACHE dos pacientes sobreviventes de 2,6 pontos e dos óbitos de 6 pontos para este procedimento.

Foi calculado o efeito da idade (valor no cálculo do APACHE II) somente sobre a mortalidade e morbidez do Grupo 2 (Tabela 5). Porém sem significância.

A partir da equação formulada por Knaus ${ }^{3}$ para predizer óbito, calculados os valores: APACHE baixo, com expectativa de mortalidade de $4 \%$; APACHE moderado, com $6 \%$ de mortalidade esperada; e APACHE alto, com $11 \%$. Utilizando o teste quiquadrado corrigido Yates para comparações de proporções, não foi achada diferença estatística entre as porcentagens da mortalidade esperada e observada. A comparação da mortalidade esperada e observada é mostrada na Figura 1. 


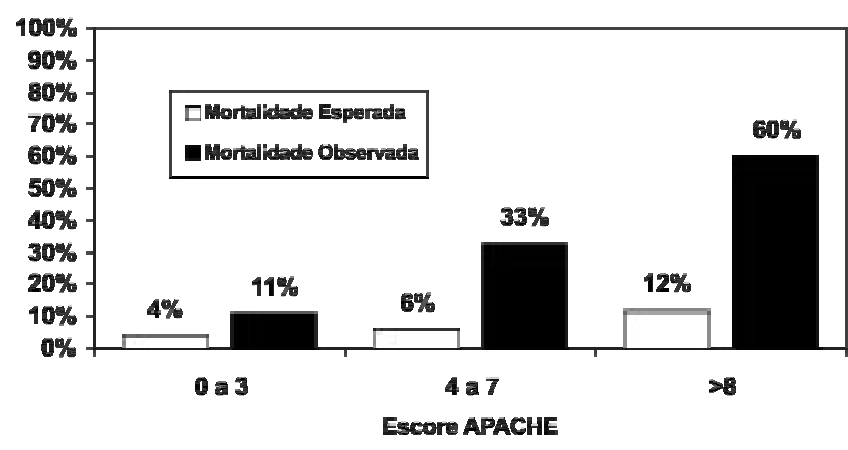

Figura 1 - Mortalidade Esperada e Observada do grupo 2.

\section{DISCUSSÃO}

O risco cirúrgico é definido como a probabilidade de surgirem complicações devidas a outras condições e doenças associadas ${ }^{13}$. É dividido em dois períodos pós-operatórios: as primeiras 48 horas, nas quais os fatores cardiovasculares e pulmonares são mais importantes, e de 3 a 30 dias, período no qual os fatores imunológicos e nutricionais desempenham um papel chave ${ }^{7}$.

A morbidade e a mortalidade pós-operatória dependem de muitos fatores. Estes poderiam ser grosseiramente divididos em fatores de agressão (estresse cirúrgico, tipo de cirurgia, anestesia, tipo de doença, infecção, neoplasia, radioterapia etc.) e fatores de defesa (estado nutricional, sistema imunológico e estado fisiológico). Estes últimos são mais difíceis de serem mensurados e, amiúde, não utilizados nas escalas de classificação de riscos ${ }^{7}$.

O APACHE é um sistema de avaliação da fisiologia aguda e da saúde crônica com 34 medidas fisiológicas e posteriormente reduzidas para 12 variáveis fisiológicas, que são mais preditivas da mortalidade, somadas a pontos para idade e para saúde crônica referido como APACHE II ${ }^{2,3}$.

Embora este sistema de escore tenha primariamente sido utilizado em pacientes de unidades de tratamento intensivo para prognosticar risco de morte, vários outros estudos têm validado este sistema em pacientes com sepse abdominal, pancreatite necrotizante aguda e abscesso pancreático, trauma grave, fístulas enterocutâneas pós-operatórias e, mais recentemente, em operações de grande porte como hepatectomias, gastrectomias eduodenopancreatectomias ${ }^{4,5,14-18}$.

Schein ${ }^{6}$ mensurou o escore APACHE II em pacientes submetidos à operação de urgência para o controle de úlcera péptica sangrante. Amostra de 154 pacientes onde 96 foram operados por ulcera gástrica e 58 por úlcera duodenal. O escore médio geral esteve em 11,6 pontos (variando de 2 a 25 pontos), sendo que os pacientes que evoluíram para o óbito tiveram média de 17,5 pontos e os que sobreviveram de 10,8 pontos. Dividiram o risco cirúrgico naqueles com escore $<11$, baixo risco, devido à inexistência de óbito; o grupo de alto risco que determinava escores de 11 a 20, que apresentou alto índice de mortalidade; finalmente, o grupo de pacientes

Tabela 3 - Morbidade do Grupo 2.

\begin{tabular}{cccc}
\hline Complicações & Número & (\%) de pacientes * \\
\hline Maiores & Sepse Abdominal & 12 & $(34)$ \\
& - Insuficiência Renal Aguda & 6 & $(35)^{* *}$ \\
& Complicação Cardíaca & 4 & $(23)$ \\
& . Insuficiência Respiratória & 2 & $(10)^{* *}$ \\
Menores & - Infecção Respiratória & 5 & $(29)^{* *}$ \\
& - Infecção Urinária & 24 & $(66)$ \\
& . Deiscência & 22 & $(53)$ \\
& - Infecção Ferida Operatória & 4 & $(10)$ \\
Nenhuma & & 9 & $(22)$ \\
\end{tabular}

* A soma do número de complicações dividida nas duas classificações ultrapassa o número geral, devido a alguns pacientes terem desenvolvido mais que uma complicação.

**Pacientes que evoluíram para óbito (seis casos de sepse abdominal, cinco casos de insuficiência respiratória por infecção respiratória e um caso de complicação cardíaca).

Tabela 4 - APACHE II pré-operatório no Grupo 2 e índices de morbidez e mortalidade.

\begin{tabular}{|c|c|c|c|c|c|c|c|c|c|}
\hline \multirow[b]{2}{*}{ APACHE II Pré-operatório } & \multicolumn{4}{|c|}{ Morbidez } & \multicolumn{5}{|c|}{ Mortalidade } \\
\hline & $\mathbf{N}$ & $\mathrm{n}$ & $\%$ & $\mathrm{n}$ & $\%$ & RR & IC & $\bar{c}$ & p \\
\hline Baixo $(0-3)$ & 27 & 19 & 70 & 3 & 11 & 1 & - & - & \\
\hline Moderado (4-7) & 18 & 12 & 66 & 6 & 33 & 3 & $(0,86-$ & $10,48)$ & $<.08$ \\
\hline Alto $(\geq 8)$ & 5 & 5 & 100 & 3 & 60 & 5,4 & $(1,49-$ & $19,51)$ & $<.04$ \\
\hline Todos & 50 & 36 & 72 & 12 & 24 & & & & \\
\hline
\end{tabular}


Tabela 5 - Efeito da idade nos índices de mortalidade e morbidez pós-operatória.

\begin{tabular}{lrrrrr}
\hline & & \multicolumn{2}{c}{ Morbidez } & \multicolumn{2}{c}{ Mortalidade } \\
\cline { 3 - 6 } Idade (anos) & \multicolumn{1}{c}{$\mathbf{N}$} & $\mathbf{n}$ & $\boldsymbol{\%}$ & $\mathbf{n}$ & $\boldsymbol{\%}$ \\
\hline$<40$ & 4 & 3 & 75 & 0 & \\
$40-59$ & 19 & 13 & 69 & 5 & 26 \\
$\geq 60$ & 27 & 19 & 70 & 7 & 25 \\
\hline
\end{tabular}

gravíssimos com pontuação superior a 20, que não apresentavam condições de qualquer intervenção cirúrgica.

Em outro estudo, Schein ${ }^{19}$ analisou pacientes acometidos de úlcera péptica perfurada. Foram 162 casos, sendo 95 casos de origem duodenal e 67 gástricos. A média do escore nos pacientes sobreviventes foi de oito e no grupo de óbito de 14,5 pontos. Quando utilizado ponto de corte de APACHE igual a 10 observou-se que todos os óbitos ocorreram no grupo com pontuação superior (35\% dos casos deste grupo). Os autores concluem que a ascensão do escore acompanha a mortalidade e demonstrou-se valor prognóstico significativo quando se dividiu a amostra em três classes: risco baixo (APACHE < 11), com 0\% de mortalidade; risco alto (APACHE entre 11 e 20), com $32 \%$ de mortalidade; e risco crítico (APACHE $>20$ ), $\operatorname{com} 75 \%$ de mortalidade.

Gagner $^{12}$ avaliou pacientes submetidos à hepatectomia direita. Foram 70 pacientes, com coleta de dados nas 24 horas que precediam a hepatectomia, todos com neoplasias. Divididos por grau de risco: escore baixo (0-3 pontos) com nenhum óbito e $34 \%$ de morbidez; escore moderado (4-7 pontos) com taxa de mortalidade de $3 \%$ e $54 \%$ de morbidez; escore alto ( 8 e maior pontuação) com $20 \%$ de mortalidade e $80 \%$ de morbidade. Os autores concluem que escores maior de 8 não teriam indicação de ressecção maior, pois prediz índices de morbidade maior de $80 \%$ e mortalidade maior de $20 \%$.

Fahn ${ }^{20}$ utilizou o APACHE II em pacientes que desenvolveram fístula esofagovisceral intratorácica, após ressecção de adenocarcinoma de cárdia gástrico. Dados de 18 (4\%) doentes. O escore foi mensurado em quatro momentos: na admissão do paciente, imediatamente no pós-operatório, no dia do diagnóstico da deiscência e semanalmente até o paciente tivesse alta hospitalar ou óbito. Foram divididos os casos em dois grupos, escore menor igual a 10 e maior deste limite. Nenhum paciente morreu no grupo com apache igual e menor de 10 e todos óbitos foram no maior de 10 , sendo estatisticamente significante $(\mathrm{p}<0,0004)$.

Goffi $^{21}$ estudou o APACHE II em pacientes submetidos à operação de grande porte para comparar sua habilidade de predizer o desfecho com a da Associação Americana de Anestesiologista - ASA. O APACHE II foi mensurado na admissão hospitalar e quando completada 24 horas do ato operatório, e o ASA somente no pré-operatório. Cento e oitenta e sete pacientes participaram do estudo, com idade média de 65 anos (mortalidade 9\%). A regressão logística evidenciou que as complicações aumentam devido ao risco operatório que é expressa pelo aumento dos escores (ambos), onde o APACHE
II teve melhor habilidade para predizer resultados que a ASA. Além disto, diferente dos outros trabalhos até agora citados, o escore medido com 24 horas da cirurgia esteve relacionado com melhor probabilidade de predizer prognóstico que o da admissão.

Instituições brasileiras já realizaram trabalhos utilizando o APACHE II. O trabalho realizado por Rizoli ${ }^{18}$, da Universidade de Campinas em 1993, utilizou-o para comparação de tratamento definitivo (vagotomia ou gastrectomia) com o tratamento paliativo (sutura) num estudo prospectivo, de pacientes acometidos de úlcera duodenal perfurada. Os pacientes tiveram como critérios de inclusão, além de curso menor de 12 horas de perfuração, sem sinais de choque e sem comorbidades associadas, teriam de ter contagem de APACHE II menor de 11. Foram 65 doentes, sendo submetidos a: 33(51\%) casos de vagotomia; 25(38\%) casos de sutura com "Patch"; e $7(11 \%)$ casos de gastrectomia. Os escores médios dos grupos foram os seguintes: Vagotomia com 1 ponto; Sutura com "patch" com cinco pontos; e gastrectomia com cinco pontos. Os autores chegaram à conclusão que a vagotomia teve melhores resultados que a simples sutura $(\mathrm{p}<.017)$ e gastrectomia $(\mathrm{p}<0,028)$, relacionados a mortalidade e morbidade.

Machado $^{22}$ et al., desenvolveram estudo sobre os fatores de risco para óbito, nas reoperações abdominais em operação de urgência, analisando-se comparativamente o diagnóstico inicial do doente, o número, a causa e a época da relaparotomia, a existência de doenças associadas, a idade e a gravidade do paciente após a primeira cirurgia. Dados de 40 pacientes, onde a mortalidade geral foi de 42,5\% (17casos). Divididos com APACHE até 10 pontos, incluindo 25 doentes e 7 óbitos ( $28 \%$ de mortalidade); maior de 10 pontos, incluindo 15 doentes e 10 óbitos (66,7\% de mortalidade). Comparativamente, escore de dez teve mortalidade significativamente superior $(\mathrm{p}<0,05)$. Ainda, mais dois fatores foram significativos, sendo a presença de doenças associadas e a idade acima de 50 anos.

O preenchimento do protocolo não trouxe dificuldades sendo de fácil utilização e entendimento. As variáveis fisiológicas necessárias (laboratoriais ou não) são de fácil obtenção, pois fazem parte de qualquer pré-operatório. A única exceção seria a gasometria arterial, com dosagem possível em poucos minutos num hospital geral.

O Grupo 1 apresentou média de 2,5 pontos e o Grupo 2 de 3,9 pontos, alcançando significância estatística, demonstrando que a doença que acomete o paciente já é uma carga de morbidade significativa.

A morbidez geral e por classes de escore, apresentou-se significativamente maior no Grupo 2. A discriminação da classificação de morbidez revela que apenas um terço (34\%) dos pacientes desenvolveu complicações maiores, com risco de vida.

Quando se estabeleceu o parâmetro da mortalidade, ressaltou-se o peso da operação de grande porte, com maior estresse cirúrgico, e principalmente, o valor da pontuação do APACHE em escores máximos. A significância estatística foi alcançada apenas na mortalidade geral e na classe de escore alto, não havendo alterações entre pacientes submetidos à operação do Grupo 1 e 2 nos escores baixo e moderado. Estes 
achados, tornam os pacientes com APACHE maior de oito críticos neste estudo, quando submetidos à operação de grande porte. Alguns trabalhos utilizaram ponto de corte sendo o limite de 10 pontos, também alcançando mortalidade significativamente maior ${ }^{6,20}$.

Demonstrou-se a tendência que o aumento das classes de escores tem em predizer aumento da mortalidade como demonstrado em outros trabalhos científicos ${ }^{4,6,14,20,21,23}$.

Uma operação compatível com contagem APACHE II pré-operatória semelhante pode resultar em prognóstico diferente, principalmente porque a agressão ou a resposta à agressão pode ser diferente. Por esse motivo, Knaus ${ }^{3}$ afirmou em seu artigo inicial sobre a contagem APACHE II que deve ser incluído um fator ponderado de acordo com o diagnóstico na internação.

Em relação aos resultados da equação para predizer mortalidade esperada versos a observada do Grupo 2 não teve diferença estatística, mesmo com as diferentes percentagens descritas, revelando que o cálculo do índice de mortalidade baseada no escore APACHE II e numeração categórica não falhou em predizer prognóstico, em conformidade a vários autores $^{21,23,24}$. Diferente do encontrado por Cerra $^{24}$ que achou diferença estatística entre os valores esperado e observado revelando a falha do APACHE II em predizer IMOS e óbito.

A avaliação fisiológica pré-operatória deve ser utilizada por dois motivos. O primeiro é que ela estabeleça o risco de um determinado paciente, e, no preparo para uma operação eletiva, deve-se atingir a contagem de APACHE II ideal ou a mais baixa possível. O segundo motivo é que a avaliação fisi- ológica padroniza um grupo de pacientes e ajuda a comparar a mesma operação ${ }^{18}$ entre cirurgiões ou instituições.

Uma nova versão do presente índice, o escore APACHE III ${ }^{25}$, tem sido apresentado como para solucionar deficiências apresentadas pelo anterior como demonstrado por Cerra $^{5}$, que esse não prediz a evolução de IMOS e conseqüente mortalidade, bem como não valoriza alterações cardiológicas ou estado nutricional do paciente, mas como demonstrado por Barie ${ }^{26}$ a última edição do escore pode tornam-se muito complexa para o uso diário.

De acordo com os resultados, o aumento do escore está relacionado com o aumento dos índices de morbidez e mortalidade. Paciente submetido à operações de grande porte por neoplasia, com escore pré-operatório de oito ou mais pontos devem ser direcionados a uma otimização de sua avaliação biofísica, com intuito da diminuição do escore, pois prediz taxas altas de morbidez e mortalidade.

\section{CONCLUSÕES}

O escore APACHE II demonstrou tendência para predizer desfecho a pacientes submetidos à operação de grande porte.

Pacientes apresentando escore APACHE II maior que oito, quando submetidos à operação de grande porte, poderão apresentar grande índice de morbidez e mortalidade.

O escore APACHE II é útil na padronização de amostra de diferentes estudos com intuito de comparação de dados.

\begin{abstract}
Background:The general objective of this work was to compare APACHE II scores referent to prognostic in the preoperative period of major and minor surgeries. Specific objectives included: (1) the evaluation of the ability of APACHE II to predict morbidity in the preoperative period; (2) the comparison of the results of both types of surgery; and (3) the comparison of the mortality rates observed and expected according to the Knaus Equation. Methods: This was a prospective cohort study, performed in 30 months. Group 1 included 30 patients, submitted to inguinal herniorraphy, representing minor surgery. Fifty cases were included in group 2, representing major surgery: 20 were submitted to esophagectomy, 20 to gastrectomy and 10 underwent cephalic duodenopancreatectomy. Results: The average preoperative score of patients in group 1 was 2.5 points (range 0 to 8 points). The APACHE II score of group 2 was in average 3.9 points, ranging from zero to 12 points (standard deviation: 2.8 points). The morbidity of the two groups, in general or as evaluated according to low, moderate or high scores, was significantly different when compared by the chi-square test ( $p<0.00001)$. General mortality and high scores, as evaluated by the Yates-corrected chi-square test, were different between the two groups ( $p<0.001$ and $p<0.0005)$. When the mortality rate was classified according to the score of points, no statistically significant differences were observed between low and moderate scores. Conclusions: The APACHE II score, when applied during the preoperative period. showed the power to predict the outcome of patients submitted to major surgeries. High morbidity and mortality rates are associated to patients presenting APACHE II scores higher than eight, particularly if submitted to major surgery.
\end{abstract}

Key words: APACHE; Prognosis; Preoperative care. Postoperative complications.

\section{REFERÊNCIAS}

1. Dripps RD, Lamont A, Eckenhoff JE. The role of anesthesia in surgical mortality. JAMA. 1961;78:261-6.

2. Knaus WA, Zimmerman JE, Wagner DP, Draper EA, Lawrence DE. APACHE - acute physiology and chronic health evaluation: a physiologically based classification system. Crit Care Med. 1981;9(8):591-7.
3. Knaus WA, Draper EA, Wagner DP, Zimmerman JE. APACHE II: a severity of disease classification system. Crit Care Med. 1985;13(10):818-29.

4. Bohnen JM, Mustard RA, Oxholm RN, Schouten BD. APACHE II score and abdominal sepsis. A prospective study. Arch Surg. 1988;123(2):225-9.

5. Cerra FB, Negro F, Abrams J. APACHE II score does not predict multiple organ failure or mortality in postoperative surgical patients. Arch Surg. 1990; 125(4):519-22. 
6. Schein M, Gecelter G. APACHE II score in massive upper gastrointestinal haemorrhage from peptic ulcer: prognostic value and potential clinical applications. Br J Surg. 1989;76(7):733-6.

7. Eubanks S. Hernias. In: Sabiston DC, editor. Textbook of surgery. The biological basis of modern surgical practice. $15^{\text {th }}$ ed. Philadelphia: WB Saunders; 1997.

8. Orringer MB. Resection of the esophagus. In: Shields TW, Locicero J, Ponn RB, editors. General thoracic surgery. $15^{\text {th }}$ ed. Philadelphia: Lippincott Willians \& Wilkins; 2000.

9. Aoki T, Takayama S. Subtotal gastrectomy for gastric cancer. In: Nyhus LM, Baker RJ, Fischer JE, editors. Mastery of surgery. $3^{\text {rd }}$ ed. Boston: Little, Brown and Company; 1997.

10. Brenan MF. Total gastrectomy for carcinoma. In: Nyhus LM, Baker RJ, Fischer JE. Editores. Mastery of Surgery. $3^{\text {rd }}$ ed. Boston: Little, Brown and Company; 1997.

11. Pezzuli G, Spina GP. Tumores del pancreas. In: Veronesi U, editor. Cirugía oncológica. Buenos Aires: Editora Médica Panamericana; 1991.

12. Gagner M, Franco D, Vons C, Smadja C, Rossi RL, Braasch JW. Analysis of morbidity and mortality rates in right hepatectomy with the preoperative APACHE II score. Surgery. 1991;110(3):487-92.

13. Lehmkuhl P, Peck-Thole S, Pichlmayr I. A new scoring system for disease intensity in a surgical intensive care unit. World $\mathbf{J}$ Surg. 1989;13(3):252-8.

14. Altomare DF, Serio G, Pannarale OC, Lupo L, Palasciano N, Memeo V, Rubino M. Prediction of mortality by logistic regression analysis in patients with postoperative enterocutaneous fistulae. Br J Surg. 1990;77(4):450-3.

15. Bohnen JMA, Mustard RA, Schouten BD. Steroids, APACHE II score, and the outcome of abdominal infection. Arch Surg. 1994;12991):33-7; discussion 37-8.

16. Marshall JB. Acute pancreatitis. A review with an emphasis on new developments. Arch Intern Med. 1993;153(10):1185-98.

17. New classification of physical status. Anesthesiology 1963; 24 : 111.

18. Rizoli SB, Neto AC, Diorio AC, Moreira MA, Mantovani M. Risk of complication in perforated duodenal ulcer operations according to the surgical technique employed. Am J Surg. 1993;59(5):312-4.
19. Schein M, Gecelter G, Freinkel Z, Gerding H. APACHE II in emergency operations for perforated ulcers. Am J Surg. 1990;159(3):309-13.

20. Fahn HJ, Wang LS, Huang MS, Hsu WH, Huang MH. Leakage of intrathoracic oesophagovisceral anastomoses in adenocarcinoma of the gastric cardia: changes in serial APACHE II scores and their prognostic significance. Eur J Surg. 1997;163(5):345-50.

21. Goffi L, Saba V, Ghiselli R, Necozine S, Mattei A, Carle F. Preoperative APACHE II and ASA scores in patients having major general surgical operations: prognostic value and potential clinical applications. Eur J Surg. 1999;165(8):730-5.

22. Machado MAC, Souza Junior AL, Poggeti RS, Utiyama EM, Branco PD, Birolini D. Fatores de risco em relaparotomia na cirurgia de urgência. Rev Hosp Clin Fac Med Univ São Paulo. 1994; 49(1):17-20.

23. Giangiuliani G, Gui D, Bonatti P, Tozzi P, Caracciolo F. APACHE II in surgical lung carcinoma patients. Chest. 1990;98(3):627-30.

24. Koperna T, Semmler D, Marian F. Risk stratification in emergency surgical patients: is the APACHE II score a reliable marker of physiological impairment? Arch Surg. 2001;136(1):55-9.

25. Knaus WA, Wagner DP, Draper EA, Zimmerman JE, Bergner M, Bastos PG, Sirio CA, Murphy DJ, Lotring T, Damiano A, et al. The APACHE III prognostic system. Risk prediction of hospital mortality for critically ill hospitalized adults. Chest. 1991;100(6):1619-36.

26. Barie PS, Hydo LJ, Fischer E. Comparison of APACHE II and III scoring system for mortality prediction in critical surgical illness. Arch Surg. 1995; 130(1):77-82.

Como citar este artigo:

Castro Júnior MAM, Castro MAM, Castro AP, Silva AL. Sistema de escores APACHE II e o prognóstico de pacientes submetidos à cirurgia de grande e pequeno porte. Rev Col Bras Cir. [periódico na internet] 2006 Set-Out; 33(5). Disponível em URL: www.scielo.br/rcbc

Endereço para correspondência:

Miguel Angelo Martins de Castro Junior

Rua Duque de Caxias, 585

Bairro: Centro.

96200-020 - Rio Grande, RS

Tel: (53) 32328988

Email:miguelacjr@hotmail.com 